\title{
Surgical Treatment for Pulmonary Metastasis of Pancreatic Ductal Adenocarcinoma: Study of 12 Cases
}

\author{
MOTOAKI YASUKAWA, TAKESHI KAWAGUCHI, NORIKAZU KAWAI, TAKASHI TOJO and SHIGEKI TANIGUCHI \\ Department of Thoracic and Cardiovascular Surgery, Nara Medical \\ University School of Medicine, Kashihara, Japan
}

\begin{abstract}
Background/Aim: Few studies have reported results with pulmonary metastasectomy for pancreatic ductal adenocarcinoma (PDAC), probably because of their extremely poor outcomes. The aim of this study was to review our institutional experience and analyze the outcomes of pulmonary metastasectomy for PDAC. Patients and Methods: A retrospective case-control study of patients undergoing pulmonary metastasectomy for isolated lung metastasis of PDAC between 2004 and 2016. Clinicopathological features were analyzed and survival curves were plotted using the Kaplan-Meier method. Results: Twelve patients were included. The median followup period after metastasectomy was 33 months. At the time of analysis, five patients had deceased, two were alive with disease, and five were alive without disease. The median survival time after pulmonary metastasectomy was 47 months. The estimated 3- and 5-year overall survival rates were $62.3 \%(95 \% C I=27.8-84.0)$ and $31.2 \%(95 \% C I=1.7$ 71.7). Conclusion: Outcomes were favorable following pulmonary metastasectomy in selected patients with PDAC.
\end{abstract}

Pancreatic ductal adenocarcinoma (PDAC) is a highly aggressive malignancy. The American Cancer Society estimated approximately 48,960 patients diagnosed with PDAC in 2015, with more than 40,560 deaths attributable to the disease (1). The median 5-year survival of $6 \%$ is likely a result of the tumor's invasiveness and propensity toward metastasis (2). Once PDAC has metastasized to distant organs, the prognosis is dismal with an overall 5 -year survival of only $1 \%(3,4)$.

This article is freely accessible online.

Correspondence to: Motoaki Yasukawa, Department of Thoracic and Cardiovascular Surgery, Nara Medical University School of Medicine, Kashihara, Nara 634-0813, Japan. Tel: +81 744223051, Fax: +81 744248040, e-mail: myasukawa@naramed-u.ac.jp

Key Words: Pulmonary metastasectomy, pancreatic ductal adenocarcinoma, prognosis.
Pulmonary metastasectomy has been shown to provide a survival benefit for colorectal cancer patients with pulmonary metastases (5). And recently, Karagkiouzis et al. (6) reported that combined surgical resection of primary non-small cell lung cancer and solitary hematogenous metastasis might be associated with improved survival. Recurrent PDAC, in comparison, has been notoriously difficult to manage surgically as it is typically characterized by aggressive growth, a multifocal pattern of recurrence, and technically unresectable disease (7). Therefore, although the lung is one of the common sites for distant metastasis in PDAC patients (8), the number of reports of pulmonary metastasis of pancreatic cancer is limited. Recently, Arnaoutakis et al. (9) reported the results of nine patients and Tagawa et al. (10) reported the results of four patients who underwent pulmonary metastasectomy for PDAC. Herein, we retrospectively review our institutional experience and analyze the outcomes of 12 patients who underwent surgery for pulmonary metastases of PDAC. To the best of our knowledge, this is the largest case-control study size reported.

\section{Patients and Methods}

This study evaluated 12 patients who underwent surgical treatment for pulmonary metastasis of pancreatic cancer and were then histologically diagnosed with PDAC between January 2004 and December 2016 at our institute. The pulmonary metastases were isolated without evidence of other sites of disease recurrence at the time of metastasectomy. The exclusion criteria for entry to the study were complications from other malignant diseases after enrollment. Thus, the lungs represented the first recurrence site of metastasis in this study. Data were collected from patient's medical charts, operative notes and pathologic examination records. Follow-up data were gathered from outpatient clinic records

Because individual patients were not identified in this retrospective study, the Ethics Review Board of our institute waived the requirement to obtain written informed consent from patients and approved the study protocol.

We used the VATS technique to resect pulmonary metastases. Fortunately, there was no need for conversion to open thoracotomy for any patient in this series. 
Table I. Details of 12 patients.

\begin{tabular}{|c|c|c|c|c|c|c|c|c|c|c|c|c|}
\hline Case & $\begin{array}{l}\text { Age } \\
(y)\end{array}$ & $\begin{array}{c}\text { Preoperative } \\
\text { therapy }\end{array}$ & Operation & $\begin{array}{c}\text { Pathologic } \\
\text { stage (UICC) }\end{array}$ & $\begin{array}{c}\text { Postoperative } \\
\text { therapy }\end{array}$ & $\begin{array}{l}\text { DFI } \\
(\mathrm{mo})\end{array}$ & $\begin{array}{l}\text { Pulmonary } \\
\text { operation }\end{array}$ & $\begin{array}{l}\text { Number of } \\
\text { lung mets }\end{array}$ & Location & $\begin{array}{l}\text { Size } \\
(\mathrm{mm})\end{array}$ & $\begin{array}{c}\text { Clinical } \\
\text { course }\end{array}$ & $\begin{array}{l}\text { SAR } \\
(\mathrm{mo})\end{array}$ \\
\hline 1 & 83 & None & PD & IIB & G & 15 & Wedge & 1 & RLL & 25 & DD & 10 \\
\hline 2 & 78 & None & PD & III & $\mathrm{HAI}+\mathrm{G}$ & 8 & Wedge & 3 & RUL,RML,RLL & $10,18,11$ & $\mathrm{AD}$ & 35 \\
\hline 3 & 64 & None & DP & IIA & $\mathrm{HAI}+\mathrm{G}$ & 42 & Wedge & 1 & LUL & 12 & AW & 33 \\
\hline 4 & 78 & $\mathrm{G}+\mathrm{RT}$ & PD & IIA & $\mathrm{HAI}+\mathrm{G}$ & 50 & Wedge & 1 & LLL & 10 & AW & 33 \\
\hline 5 & 72 & $\mathrm{G}+\mathrm{RT}$ & DP & IIB & $\mathrm{HAI}+\mathrm{G}$ & 39 & Wedge & 2 & RUL,RML & 20,10 & AW & 33 \\
\hline 6 & 75 & None & Inoperable & IV & $\mathrm{G}+\mathrm{S}+\mathrm{RT}$ & 49 & Lobectomy & 1 & RUL & 28 & DD & 24 \\
\hline 7 & 77 & $\mathrm{G}+\mathrm{S}+\mathrm{RT}$ & DP & III & $\mathrm{G}$ & 11 & Wedge & 2 & LUL,LLL & 8,12 & DD & 12 \\
\hline 8 & 77 & None & PD & IIA & $\mathrm{HAI}+\mathrm{G}$ & 74 & Lobectomy & 1 & RUL & 8 & DD & 47 \\
\hline 9 & 63 & $\mathrm{G}+\mathrm{RT}$ & PD & IIA & $\mathrm{HAI}+\mathrm{G}$ & 24 & Wedge & 1 & RLL & 17 & AW & 66 \\
\hline 10 & 70 & $\mathrm{G}+\mathrm{RT}$ & PD & III & $\mathrm{HAI}+\mathrm{G}$ & 30 & Wedge & 3 & RLL & $12,6,5$ & DD & 31 \\
\hline 11 & 80 & $\mathrm{G}+\mathrm{RT}$ & DP & III & $\mathrm{HAI}+\mathrm{G}$ & 34 & Wedge & 1 & RLL & 23 & AW & 28 \\
\hline 12 & 74 & None & DP & IA & $\mathrm{HAI}+\mathrm{G}$ & 22 & Wedge & 1 & RLL & 10 & $\mathrm{AD}$ & 6 \\
\hline
\end{tabular}

G: Gemcitabine; S: TS-1; RT: radiation therapy; HAI: hepatic arterial infusion chemotherapy; PD: pancreatoduodenectomy; DP: distal pancreatectomy; DFI: disease-free interval; wedge: wedge resection; mets: metastases; RUL: right upper lobe; RML: right middle lobe; RLL: right lower lobe; LUL: left upper lobe; LLL: left lower lobe; DD: died of disease; AD: alive with disease; AW: alive and well; SAR: survival after relapse.

Follow-up examinations were symptom oriented, but all patients received medical checkups and chest $\mathrm{X}$-rays at least twice a year, while whole-body computed tomography (CT) scans were performed at least once a year.

The disease-free interval (DFI) was defined as the period between curative primary site resection or complete remission and the first detection of pulmonary nodules. Survival curves were plotted using the Kaplan-Meier method. All analyses were conducted using EZR on R commander, version 1.33 (R Foundation for Statistical Computing, Saitama, Japan).

\section{Results}

A total of 12 patients were included. The median follow-up period after metastasectomy was 33 months (range=6-72 months). Table I shows the clinicopathological characteristics of the patients. Six patients were male and six were female. The average age on undergoing pulmonary metastasectomy was 74.25 years (range $=63-83$ years). Six patients had received induction chemoradiotherapy before primary pancreas surgery. The primary tumor had been completely resected in 11 patients, all of whom received chemotherapy after surgery. However, one patient had been inoperable at the primary site upon the first medical examination and received chemoradiotherapy until complete remission. In all 12 patients, there was no recurrence until the pulmonary metastases were revealed. All patients achieved complete resection, with no postoperative deaths or complications, and all received chemotherapy after the pulmonary metastasectomy.

At the time of analysis five patients had deceased, two patients were alive with disease, and five patients were alive without disease. The median survival time after pulmonary metastasectomy was 47 months (range=6-66 months). The estimated 3-year and 5-year overall survival rates were $62.3 \%$ (95\% confidence interval (CI) 27.8-84.0) and $31.2 \%$ (95\% CI=1.7-71.7) (Figure 1). The median survival time after primary site surgery or complete remission was 121 months, and the estimated 5-year overall survival was $82.5 \%$ (95\%CI=46.1-95.3) (Figure 2).

\section{Discussion}

Metastasectomy is considered a beneficial treatment for patients with pulmonary metastases whose primary tumor has been well controlled, and pulmonary resection has been shown to be beneficial for patients with resectable and isolated pulmonary metastases (6). However, few studies have reported such results for pulmonary metastasectomy for PDAC, probably because of their extremely poor outcomes. Among long-term survivors after pancreas resection for PDAC, the most common site for disease recurrence is the lung (11).

Arnaoutakis et al. (9) reported the results of nine patients who underwent pulmonary metastasectomy for PDAC. They showed that pulmonary metastasectomy can be performed smoothly and may be a beneficial procedure, and reported median overall survival from pancreatectomy of 51 months in patients who underwent metastasectomy and 23 months in those who had pulmonary metastasis but did not undergo metastasectomy. Moreover, the median survival after relapse was 18.6 months $(95 \% \mathrm{CI}=5.6-29.2)$ for the pulmonary metastasectomy group and 7.5 months $(95 \% \mathrm{CI}=3.4-22)$ for the control group. Our results compare favorably with these findings, the median overall survival from pancreatectomy being 121 months and the median survival after relapse 47 months. 


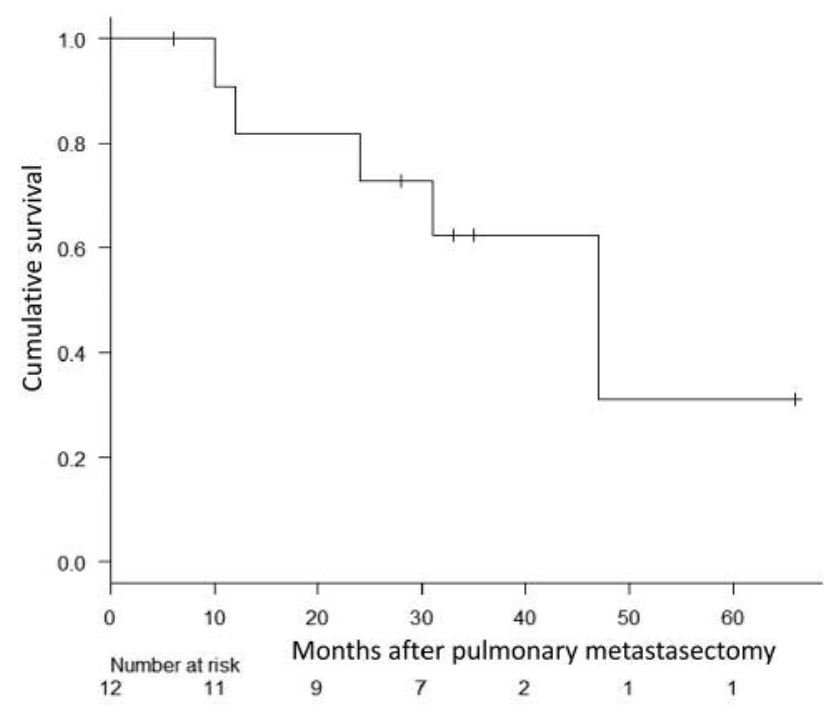

Figure 1. Overall survival of 12 patients after pulmonary metastasectomy. The median survival time was 47 months and the 5-year survival was $31.2 \%$.

It should be emphasized that this is a highly selected group of patients who developed metachronous isolated pulmonary metastasis following pancreatic resection. A relatively long interval between initial resection of primary pancreatic cancer and relapse, isolated and stable disease over time, and favorable response to systemic therapy were considered indicative of favorable pathology and a requisite to be considered for pulmonary metastasectomy.

Katz et al. (11) reported that the median overall survival was significantly longer for patients with isolated pulmonary recurrence (40.3 months) than with other metastases (20.9 months; hazard ratio 5.85, $p=0.0156$ ). Thomas et al. (7) reported their experience with 21 selective resections of solitary metastases, including seven lung metastases. Among patients who underwent reoperation, overall survival was significantly longer in those with a median first DFI of $>20$ months than in those with a first DFI of $<20$ months $(p=0.033)$.

All patients in the present study had isolated pulmonary recurrence at first relapse. Of the three patients who had a first DFI of $<20$ months, two deceased before the end of the observation period.

Selected patients with isolated pulmonary recurrence of PDAC after a prolonged DFI are good candidates to achieve a favorable prognosis through pulmonary metastasectomy. Although the lung is a major site of recurrence in PDAC, there are not many patients who have only a solitary lung metastasis. And so, it remains unclear why some patients have only a solitary lung metastasis and why patients with

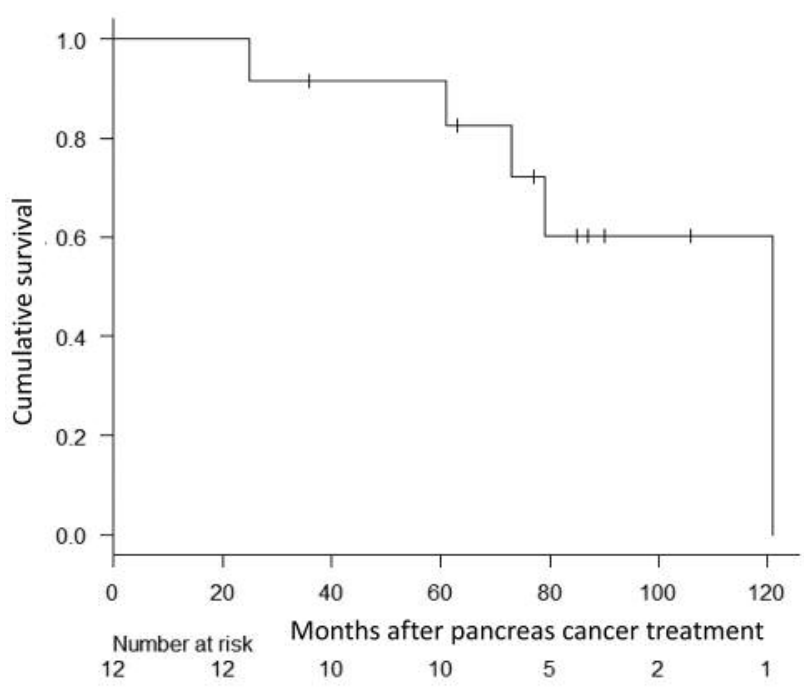

Figure 2. Overall survival of 12 patients after primary-site surgery or complete remission was 121 months and the estimated 5-year overall survival was $82.5 \%$.

metastasis to the lung as their first site of recurrence have a better prognosis. The animal models are very useful for cancer investigation. In an experimental lung-metastasis model of nude mice, Tome et al. (12) reported that tumor emboli were observed in pulmonary vessels and invading cancer cells were observed at the margin of tumor emboli eight weeks after cancer-cell implantation into the tibia. Sundaram et al. (13) reported that high-fat diet significantly increased the number of lung metastases due to the increase of the abundance of proinflammatory cytokines and angiogenic factors in plasma and tumors. There might be some differences between solitary lung metastasis and not concerning cancer-cell adaptation to the pulmonary microenvironment and tumor progression of pulmonary metastasis.

There are two main limitations to this study, namely its retrospective design and small sample size. More specifically, the patients in this study are highly selected and have good biological tumor characteristics identified by a favorable response to systemic therapy, and our patients undergoing pulmonary metastasectomy had a relatively long interval between initial pancreatectomy and pulmonary relapse.

\section{Conclusion}

We report favorable outcomes following pulmonary metastasectomy in patients who had undergone pancreatic resection for PDAC, although for this small retrospective series we acknowledge the aforementioned limitations. A study involving a large, multi-institutional cohort will be 
important to validate these findings. Further investigation is required to determine which patients would be best suited for these interventions.

\section{Conflicts of Interest}

The Authors declare that they have no conflict of interest in regard to this study.

\section{References}

1 Siegal RL, Miller KD and Jemal A: Cancer statistics, 2015. CA Cancer J Clin 65: 5-29, 2015.

2 DeSantis CE, Lin CC, Mariotto AB, Siegel KD, Kramer JL, Alteri R, Robbins AS and Jemal A: Cancer treatment and survivorship statistics, 2014. CA Cancer J Clin 64: 252-271, 2014.

3 Lillemoe KD, Kaushal S, Cameron JL, Sohn TA, Pitt HA and Yeo CJ: Distal pancreatectomy: indications and outcomes in 235 patients. Ann Surg 229: 693-698, 1999.

4 Yeo CJ, Abrams RA, Grochow LB, Sohn TA, Ord SE, Hruban RH, Zahurak ML, Dooley WC, Coleman J, Sauter PK, Pitt HA, Lillemoe KD and Cameron JL: Pancreaticoduodenectomy for pancreatic adenocarcinoma: postoperative adjuvant chemoradiation improves survival. A prospective, single-institution experience. Ann Surg 225: 621-633, 1997.

5 Lumachi F, Chiara GB, Tozzoli R, Del Conte A and Basso SM: Factors affecting survival in patients with lung metastases from colorectal cancer. A short meta-analysis. Anticancer Res 36: 1319, 2016.

6 Karagkiouzis G, Spartalis E, Moris D, Patsouras D, Athanasiou A, Karathanasis I, Verveniotis A, Konstantinou F, Kouerinis IA, Potaris K, Dimitroulis D and Tomos P: Surgical management of non-small cell lung cancer with solitary hematogenous metastases. In Vivo 31: 451-454, 2017.

7 Thomas RM, Truty MJ, Nogueras-Gonzalez GM, Fleming JB, Vauther JN, Pisters PW, Lee JE, Rice DC, Hofstetter WL, Wolff RA, Varadhachary GR, Wang $\mathrm{H}$ and Katz MH: Selective reoperation for locally recurrent or metastatic pancreatic ductal adenocarcinoma following primary pancreatic resection. J Gastrointest Surg 16: 1696-1704, 2012.
8 Embuscado EE, Laheru D, Ricci F, Yun KJ, de Boom Witzel S, Seigel A, Flickinger K, Hidalgo M, Bova GA and LacobuzioDonahue CA: Immortalizing the complexity of cancer metastasis: genetic features of lethal metastatic pancreatic cancer obtained from rapid autopsy. Cancer Biol Ther 4: 548-554, 2005.

9 Arnaoutakis GJ, Rangachari D, Laheru DA, Iacobuzio-Donahue CA, Hruban RH, Edil BH, Pawlik TM, Schulick RD, Cameron JL, Meneshian A, Yang SC and Wolfgang CL: Pulmonary resection for isolated pancreatic adenocarcinoma metastasis: an analysis of outcomes and survival. J Gastrointest Surg 15: 1611-1617, 2011.

10 Tagawa T, Ito K, Fukuzawa K, Okamoto T, Yoshimura A, Kawasaki T, Masuda T, Iwaki K, Terashi T, Okamoto M, Shiromizu A, Motohiro A and Maehara Y: Surgical resection for pulmonary metastasis from pancreatic and biliary tract cancer. Anticancer Res 37: 1413-1416, 2017.

11 Katz MH, Wang H, Fleming JB, Sun CC, Hwang RF, Wolff RA, Varadhachary G, Abbruzzese JL, crane CH, Krishnan S, Vauthey JN, Abdalla EK, Lee JE, Pisters PW and Evans DB: Long-term survival after multidisciplinary management of resected pancreatic adenocarcinoma. Ann Surg Oncol 16: 836-847, 2009.

12 Tome Y, Yano S, Sugimoto N, Mii S, Uehara F, Miwa S, Bouvet $\mathrm{M}$, Tsuchiya H, Kanaya $\mathrm{F}$ and Hoffman RM: Use of $\alpha v$ integrin linked to green fluorescent protein in osteosarcoma cells and confocal microscopy to image molecular dynamics during lung metastasis in nude mice. Anticancer Res 36: 3811-3816, 2016.

13 Sundaram S and Yan L: High-fat diet enhances mammary tumorigenesis and pulmonary metastasis and alters inflammatory and angiogenic profiles in MMTV-PyMT mice. Anticancer Res 36: 6279-6288, 2016. 